\title{
Substrate Current and Its Correlation with Degradation of Poly-Si Thin Film Transistors
}

\author{
Lei $\mathrm{LU}^{1}$, *Mingxiang WANG ${ }^{1}$, Man $\mathrm{WONG}^{2}$ \\ ${ }^{1}$ Dept. of Microelectronics, Soochow University, China \\ 2 Dept. of Electronic and Computer Engineering, the Hong Kong Univ. of Science and Technology, Hong Kong \\ *Phone: +86-512-6915 7217 Fax:+86-512-6787 1211 Email: Mingxiang_wang@suda.edu.cn
}

\begin{abstract}
Substrate current $\left(I_{s u b}\right)$ in poly-Si thin film transistors (TFTs) is first investigated by considering their specific substrate contact configuration. When the substrate bias is very small, $I_{s u b}$ is driven by the recombination process in the channel region and is related to the trap density $\left(D_{t}\right)$ wherein. Controlled by the gate bias $\left(V_{g}\right)$, the recombination region varies from the whole channel area to a localized region near the drain, source or substrate terminal. Based on such observation, $I_{s u b}$ is used to monitor the hot carrier degradation in TFTs. The $I_{s u b}-V_{g}$ curve is applied to sensitively reflect the location of the hot carrier-induced damage region and evaluate the local $D_{t}$ increase wherein.
\end{abstract}

\section{INTRODUCTION}

Low temperature poly-Si thin-film transistor (poly-Si TFT) is a promising candidate for active-matrix displays with integrated peripheral circuits on the same glass panel [1]. It is important to characterize the poly-Si TFT itself as well as its degradation. Although the substrate current $\left(I_{\text {sub }}\right)$ in MOSFETs is commonly employed to monitor the lateral electric field which directly causes the crucial hot carrier (HC) degradation [2], the application of $I_{\text {sub }}$ in poly-Si TFTs was rarely proposed [3]. Until now, neither the mechanism of $I_{s u b}$ nor its correlation with the degradation has been clarified. In this paper, by considering specific substrate contact configuration in poly-Si TFTs, $I_{\text {sub }}$ as a function of the gate bias $\left(V_{g}\right)$ and substrate bias $\left(V_{s u b}\right)$ is investigated. At small $V_{s u b}, I_{s u b}$ is driven by the recombination process and therefore corresponds to the trap density $\left(D_{t}\right)$. Besides, controlled by $V_{g}$, the recombination area locates at the whole channel or its some parts. Finally, the $I_{s u b}-V_{g}$ characteristic is applied to accurately locate the $\mathrm{HC}$ damage region and sensitively detect the $D_{t}$ increase wherein, which is consistent with the proposed mechanism of the $\mathrm{HC}$ effect.

\section{RESULTS AND DISCUSSIONS}

\section{A. Substrate Current in poly-Si TFTs}

Shown in Fig.1 are planar (a) and cross-sectional (b) views of an $\mathrm{n}$ channel poly-Si TFT with $W / L=25 / 10 \mu \mathrm{m}$ and $W_{\text {sub }} / L_{\text {sub }}=1.6 / 6 \mu \mathrm{m}$. Poly-Si channel layer was formed by solution-based metal-induced crystallization [4] of a-Si at $630^{\circ} \mathrm{C}$. The flat band voltage $\left(V_{f b}\right)$ and the threshold voltage $\left(V_{t h}\right)$ are 0 and $10 \mathrm{~V}$, respectively.

First, the substrate contact structure is examined. As shown in Fig.1a, different from that of MOSFETs, in poly-Si TFTs substrate terminal is a $\mathrm{p}^{+}$-diffused side contact to the floating poly-Si channel. When $I_{s u b}$ is measured, the source and drain are
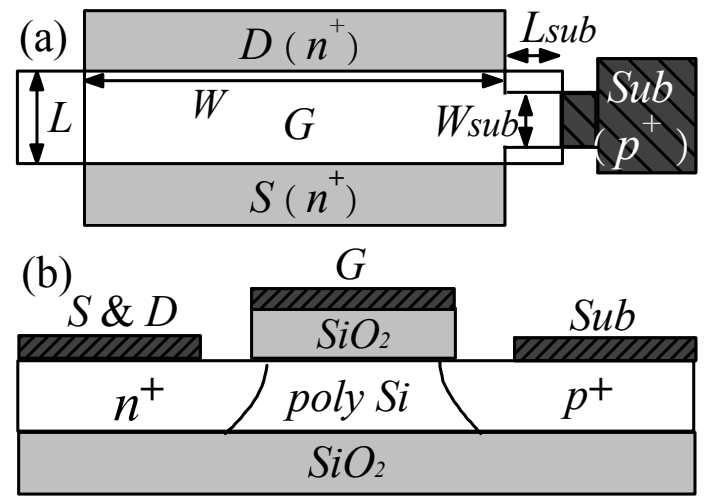

Fig. 1. Plane (a) and cross-sectional (b) views of poly-Si TFTs.

grounded while the substrate and gate terminals are biased. At the moment, carriers transport between the $\mathrm{p}^{+}$substrate contact and the $\mathrm{n}^{+}$-diffused source or drain, through an intrinsic poly-Si body. Thus $I_{s u b}$ is a diode current of a lateral PIN structure [4], with the source and drain as two parallel n-terminals and the substrate contact as the p-terminal. To be more precise, $I_{s u b}$ is the current of a gated PIN diode [5], since the intrinsic poly-Si channel is controlled by the gate, as shown in Fig. 1 b. Apparently, $I_{s u b}$ consists of two current components $I_{s u b, d}$ and $I_{s u b, s}$ from the drain and source, respectively.

In a PIN structure, the difference in Fermi level $\left(E_{f}\right)$ between the $\mathrm{p}^{+}$and $\mathrm{n}^{+}$terminals forms a potential barrier in the intrinsic region. At the $\mathrm{p}^{+}$substrate terminal, a positive $V_{\text {sub }}$ reduces the potential and therefore $I_{\text {sub }}$ is a forward current of the PIN diode which includes both diffusion and recombination currents [6-7]. As shown in Fig.2, $I_{\text {sub }}$ as a function of $V_{\text {sub }}$ are plotted in both linear and logarithmic scales. Not surprisingly, the $I_{s u b}-V_{s u b}$ curve is very similar to the typical forward current of a normal PIN diode [8-9].

As shown in Fig.2, the $I_{s u b}-V_{\text {sub }}$ curve follows a well-defined exponential characteristic described by $[4,10]$

$$
I_{\text {sub }}=I_{s} \exp \left(q V_{\text {sub }} / m k T\right)
$$

in which $I_{s}, q, m, k$ and $T$ are the saturation current, the electron charge, the ideality factor, the Boltzman constant and temperature, respectively. When $V_{\text {sub }}$ is high (e.g., $2 \mathrm{~V}$ ), the potential barrier in the channel region is significantly reduced and thus most carriers can diffuse into the doped regions [6-7]. Therefore, $I_{s u b}$ is dominated by the diffusion current and in accordance with the channel carrier density. In contrast, if $V_{s u b}$ is very small (e.g., 0.07V), the barrier is not significantly lowered and most carriers are recombined in the channel region before 


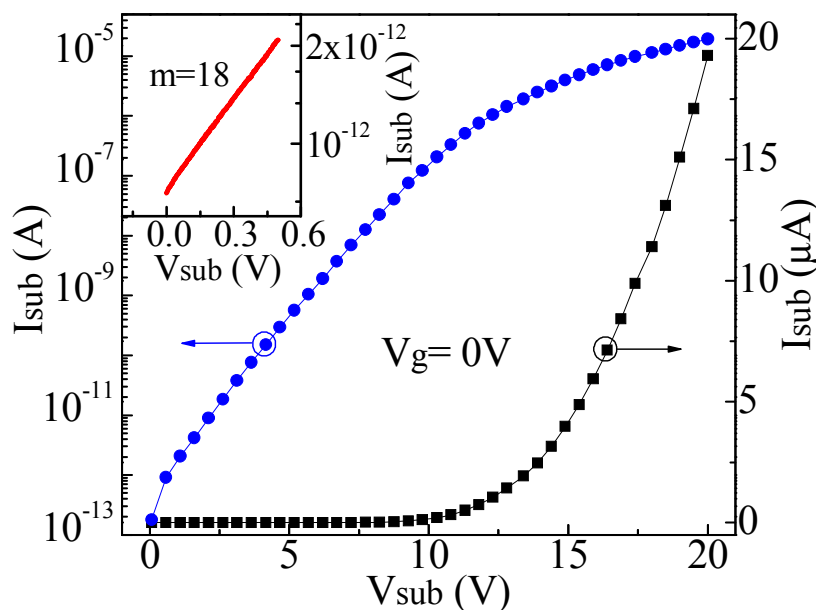

Fig. 2. The $I_{s u b}-V_{\text {sub }}$ curve measured at $V_{g}=0$ is plotted in both linear and semi-log scales. Besides, the curve in small $V_{\text {sub }}$ range is replotted in semi-log scale, as shown in the inset.

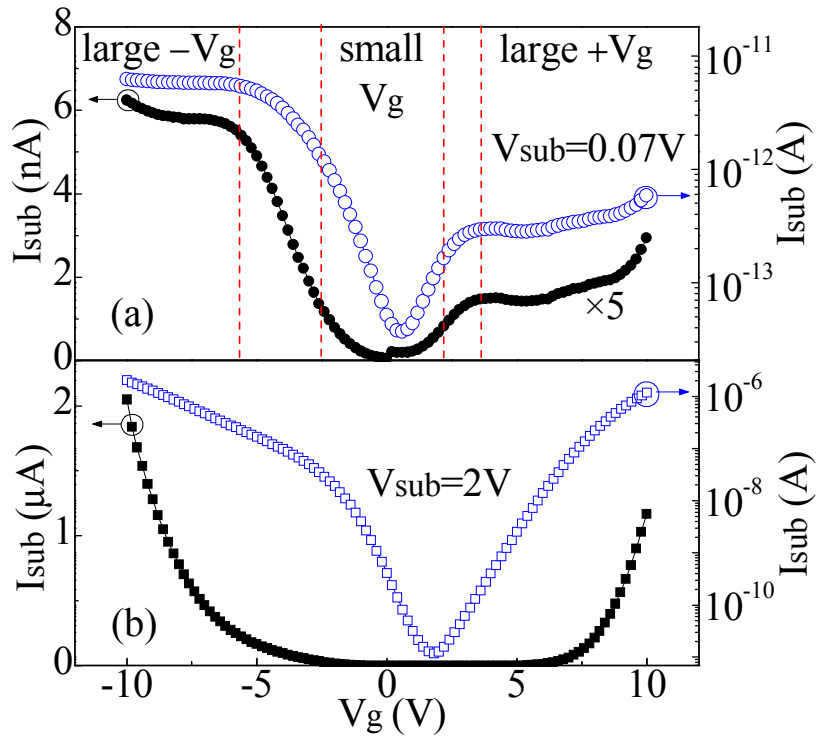

Fig. 3. The $I_{s u b}-V_{g}$ curves measured at $V_{s u b}=0.07 \mathrm{~V}$ (a) and $V_{s u b}=2 \mathrm{~V}$ (b) are plotted in both linear and semi-log scales. Besides, when $V_{g}>0, I_{s u b}$ at $V_{s u b}=0.07 \mathrm{~V}$ is magnified by five times in the linear scale.

they can diffuse into the doped electrodes [6-7]. Thus, $I_{s u b}$ is controlled by the recombination process in the intrinsic region. As shown in the inset of Fig.2, the extracted $m$ is far large than 2, which also strengthens the recombination nature of the current $[4,10]$.

In Fig. $3 \mathrm{a}$ and $3 \mathrm{~b}, I_{s u b}-V_{g}$ curves are plotted in linear and semi-log scales, measured at $V_{\text {sub }}=0.07$ and $2 \mathrm{~V}$, respectively. In small $V_{g}$ range, both curves have a similar minimum $I_{s u b}$ around $V_{g}=V_{f b}$ and $I_{s u b}$ increases exponentially with $V_{g}$. In large $\pm V_{g}$ range, $I_{s u b}$ at $V_{s u b}=0.07 \mathrm{~V}$ gradually saturates and increases slowly, while $I_{\text {sub }}$ at $V_{\text {sub }}=2 \mathrm{~V}$ does not saturate and still increases sharply. This discrepancy is due to the aforementioned different origins of $I_{\text {sub }}$ depending on $V_{\text {sub }}$.

On the one hand, $I_{s u b}$ at $V_{\text {sub }}=2 \mathrm{~V}$ is a diffusion current and in proportional to the channel carrier density, which exponentially depends on $V_{g}$. Thus, $I_{s u b}$ also increases exponentially with $V_{g}$ as shown in Fig.2b. While $I_{\text {sub }}$ at $V_{\text {sub }}=0.07 \mathrm{~V}$ is a recombination current. Taking the $-V_{g}$ range for example, the recombination current can be estimated by $[4,10]$

$$
I_{\text {sub }} \propto D_{t} A_{r} p \exp \left(q V_{\text {sub }} / m k T\right)
$$

in which $D_{t}, A_{r}$ and $p$ are respectively the trap state density, area of the recombination region, and the channel hole density. As shown in Fig.4, the $I_{s u b}-V_{\text {sub }}$ curves at different $V_{g}$ s agree well with Eq.2. Besides, $m » 2$ further confirms the recombination mechanism $[4,10]$. In Eq.2, not only $p$ increases exponentially with $V_{g}$, but also $A_{r}$ greatly depends on $V_{g}$. In other words, $V_{g}$ controls $I_{s u b}$ mainly by affecting $p$ and $A_{r}$.

As for $A_{r}$, the circumstance is complicated. In the small $-V_{g}$ range, the channel is nearly intrinsic and the potential between the $\mathrm{p}^{+}$and $\mathrm{n}^{+}$terminals drops all along the channel. Therefore, $A_{r}$ is always the whole channel region as shown in Fig.5a. Thus, in small $-V_{g}$ range, $I_{s u b}$ exponentially increases with $V_{g}$. In the large $-V_{g}$ range, the channel is in p-type and forms conductive path connecting the $\mathrm{p}^{+}$substrate. Thus PN junctions are formed near the drain and source, as indicated as $D$ and $S$ junctions in Fig. 5 b. In this case, $A_{r}$ becomes the area of the two junctions. Therefore, in large $-V_{g}$ range, although $p$ still increases with $V_{g}, A_{r}$ dramatically decreases from the whole channel region to the two junction regions. This explains why $I_{s u b}$ with $V_{s u b}=0.07 \mathrm{~V}$ first

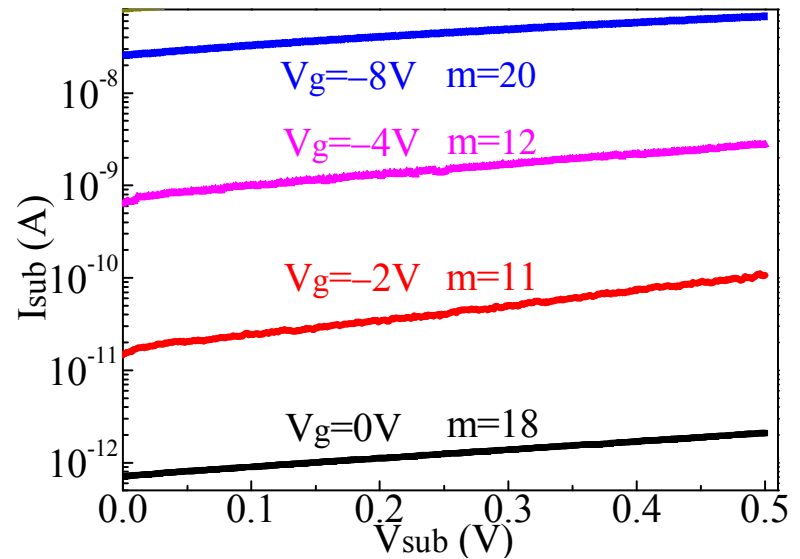

Fig. 4. The $I_{s u b}-V_{b}$ curves measured at different $V_{g}$, from which $m$ is extracted.
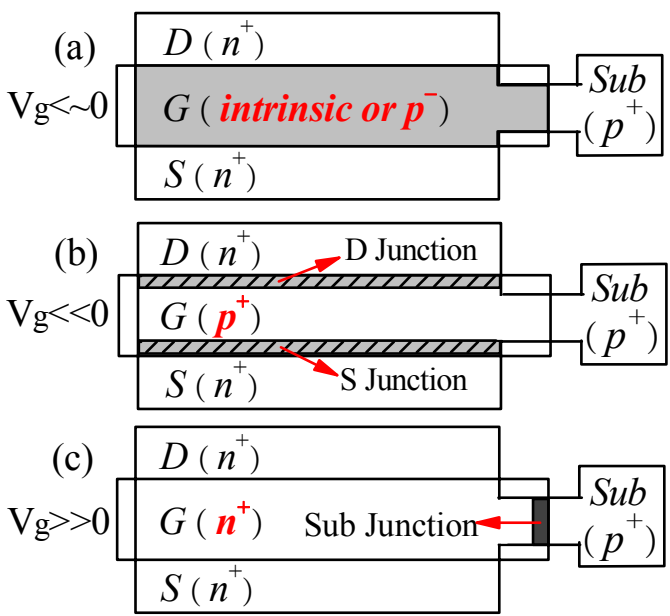

Fig. 5. The schematic diagrams of the plane views of poly-Si TFTs under various $V_{g}$ circumstances: (a) $V_{g}<\sim 0$; (b) $V_{g}<<0$; (c) $V_{g}>>0$. The shadow regions indicate the recombination areas for $I_{s u b}$ measured at small $V_{s u b}$. 
exponentially increases with $V_{g}$ but becomes saturated or even drops a little as $V_{g}$ increases further, as shown in Fig.3a. In the $+V_{g}$ range, similar mechanism occurs, except that under the high $+V_{g}, A_{r}$ shrinks more dramatically, i.e., from the whole channel region to the small "sub-junction" area, as indicated in Fig. $5 \mathrm{c}$.

As for the $I_{s u b}-V_{g}$ curve at $V_{s u b}=0.07 \mathrm{~V}$, it has not gone unnoticed that $I_{s u b}$ in the $+V_{g}$ range saturates at a much smaller level than that for $-V_{g}$ range, which also derives from the $V_{g}$ dependence of $A_{r}$. $A_{r}$ at large $+V_{\text {sub }}$ is the area of the Sub junction, while $A_{r}$ at large $+V_{\text {sub }}$ is the area of the $\mathrm{D}$ and $\mathrm{S}$ junctions, as shown in Fig.5. The former is much smaller than the later, because the width of the Sub junction (i.e., $W_{\text {sub }}$ ) is dramatically smaller than that of the $\mathrm{D}$ or $\mathrm{S}$ junction (i.e., $W$ ), as shown in Fig. 1a. Therefore, according to Eq.2, $\underline{I}_{s u b}$ in the large $-V_{g}$ range is notably smaller that that of large $+V_{g}$, as shown in Fig.3a.

\section{B. Correlation between $I_{\text {sub }}$ and HC Degradation}

Shown in Fig.6 is the time evolution of the transfer characteristic under a typical $\mathrm{HC}$ stress with $V_{g}=12 \mathrm{~V}$ and $V_{d}=20 \mathrm{~V}$ [11]. Not surprisingly, the transfer curve typically degrades with $V_{t h}$ increasing and $\mathrm{ON}$-current $\left(I_{o n}\right)$ decreasing (as indicated by the red arrow), while the subthreshold region is little affected. This is ascribed to that energetic HCs are generated by the high electric field near the drain during $\mathrm{HC}$ stress, and cause damage (or $D_{t}$ increase) to grain boundaries (GBs) and/or $\mathrm{Si}_{-} \mathrm{SiO}_{2}$ interface therein [11].

$I_{\text {sub }}-V_{\text {sub }}$ curves at $V_{\text {sub }}$ of both $2 \mathrm{~V}$ and $0.07 \mathrm{~V}$ are measured during $\mathrm{HC}$ degradation. However, the $I_{s u b}-V_{g}$ curve at $V_{s u b}=2 \mathrm{~V}$ almost does not change under HC stress, As shown in the Fig.6 inset. It is because that such $I_{\text {sub }}$ is a diffusion current and is not sensitive to $D_{t}$ variation. In contrast, the $I_{s u b}-V_{g}$ curve at $V_{\text {sub }}=0.07 \mathrm{~V}$ does change with HC stressing, as shown in Fig.7. $I_{s u b}$ at $V_{s u b}=0.07 \mathrm{~V}$ is a recombination current and in proportional to $D_{t}$ according to Eq.2. More Detailedly, $I_{s u b}$ in the small $V_{g}$ range only changes a little, indicating that $D_{t}$ in the whole channel region does not vary much. In the high $+V_{g}$ range, $I_{s u b}$ is not visibly affected by the $\mathrm{HC}$ stress either, suggesting that $D_{t}$ in the sub junction area does not change. While in high $-V_{g}$ range, $I_{\text {sub }}$ continuously increases with stress time, suggesting that the

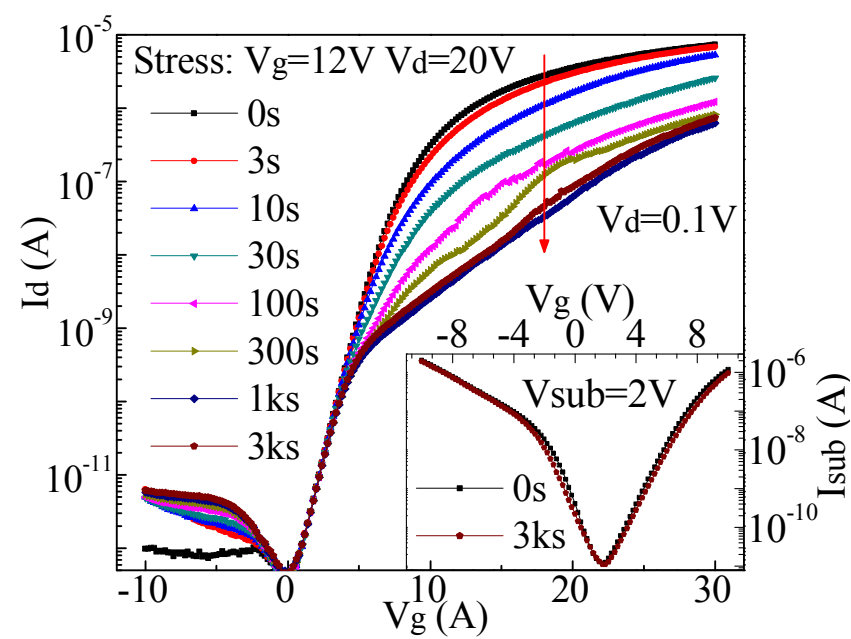

Fig. 6. The time evolutions of the transfer characteristic with $V_{g}=0.1 \mathrm{~V}$ and the $I_{s u b}-V_{g}$ curve with $V_{\text {sub }}=2 \mathrm{~V}$ under typical HC stress with $V_{g}=12 \mathrm{~V}, V_{d}=20 \mathrm{~V}$.

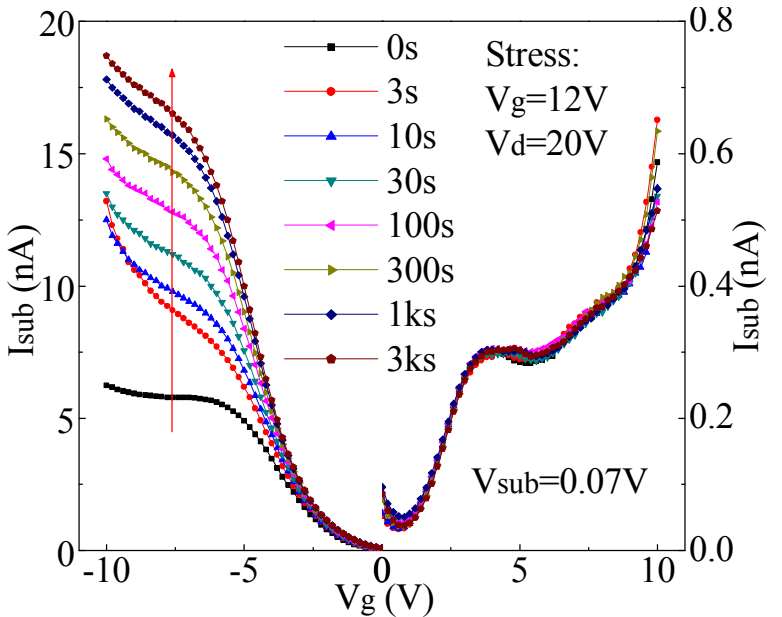

Fig. 7. The time evolution of $I_{s u b}-V_{g}$ curve at $V_{s u b}=0.07 \mathrm{~V}$ under the HC stress with $V_{g}=12 \mathrm{~V}, V_{d}=20 \mathrm{~V}$.

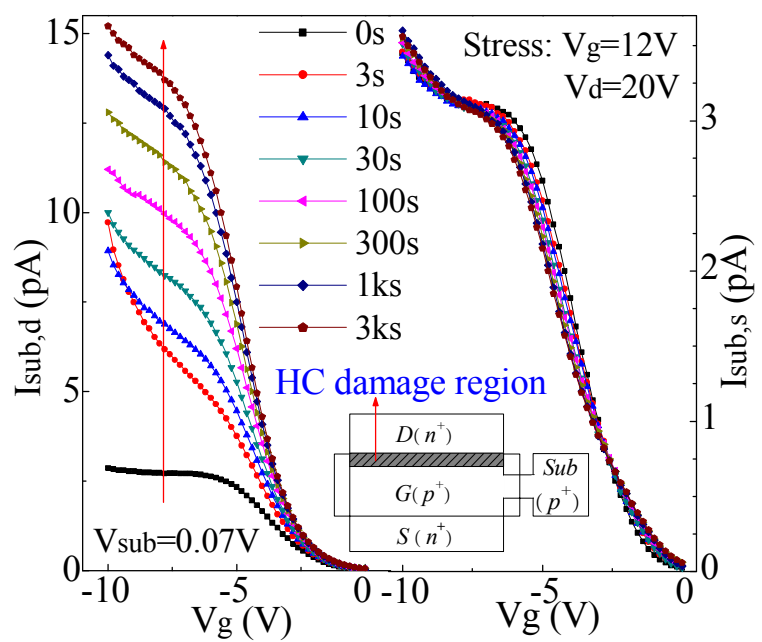

Fig. 8. The time evolutions of $I_{s u b, d^{-}} V_{g}$ and $I_{s u b, s^{-}} V_{g}$ curves with $V_{s u b}=0.07 \mathrm{~V}$ under the HC stress with $V_{g}=12 \mathrm{~V}, V_{d}=20 \mathrm{~V}$. Besides, the $\mathrm{HC}$ damage region is shown in the inset.

$D_{t}$ increases in the $\mathrm{S}$ junction and/or $\mathrm{D}$ junction. In order to further locate the region where $D_{t}$ increases, the stress time evolutions of the two $I_{s u b}$ components, i.e., $I_{s u b, d}$ and $I_{s u b, s}$ are compared in Fig.8. It is interesting that $I_{s u b, d}$ continuously increases in high $-V_{g}$ range while $I_{s u b, d}$ almost keep unaffected. Therefore, $I_{s u b}$ increase totally originates from the $I_{s u b, d}$ increase, which clearly indicates that $D_{t}$ increase or the $\mathrm{HC}$ damage occurs only near the drain junction, as shown in the inset of Fig.8. This is well consistent with previous studies [11].

It would much desirable to evaluate the $D_{t}$ increase in the $\mathrm{HC}$ damage region. The charge pumping (CP), as a generally accepted characterization method of $D_{t}$ [12], has been successfully optimized in poly-Si TFTs and applied to reliably determine $D_{t}$ [13]. Also, the $\mathrm{CP}$ has been employed to investigate the degradation of TFTs [14]. As shown in Fig.9, the $\mathrm{CP}$ current $\left(I_{c p}\right)$ is little affected under the $\mathrm{HC}$ stress with $V_{g}=12 \mathrm{~V}$ and $V_{d}=20 \mathrm{~V}$. The slight reduction of $I_{c p}\left(\Delta I_{c p}\right)$ indicates a small $D_{t}$ decrease, which is undoubtedly in contradiction with the notable degradation of $I_{o n}\left(\Delta I_{o n}\right)$, as shown Fig.10. It suggests that the $\mathrm{CP}$ has difficulties in evaluating the $\mathrm{HC}$ 


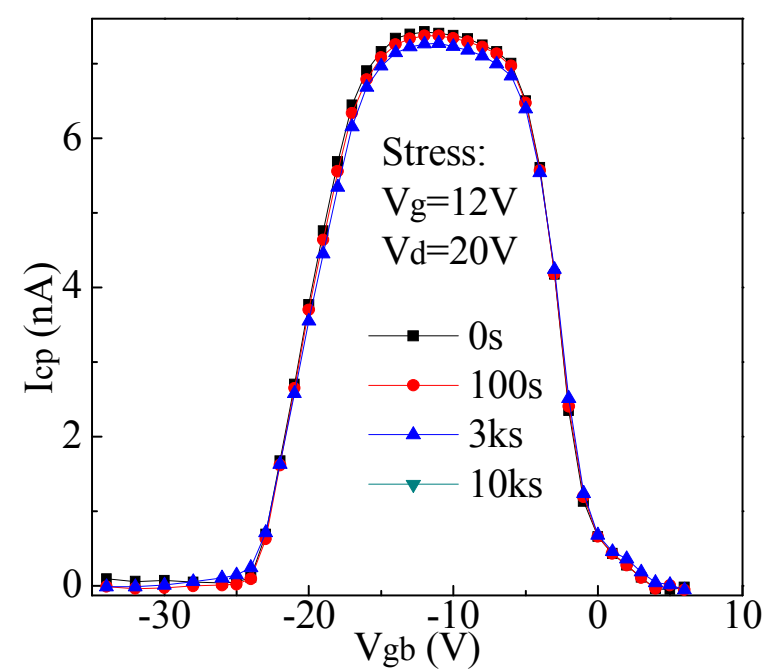

Fig. 9. The time evolutions of the CP curve, i.e., $I_{c p}$ versus the base voltage of gate pulse $\left(V_{g b}\right)$ under HC stress with $V_{g}=12 \mathrm{~V}, V_{d}=20 \mathrm{~V}$.

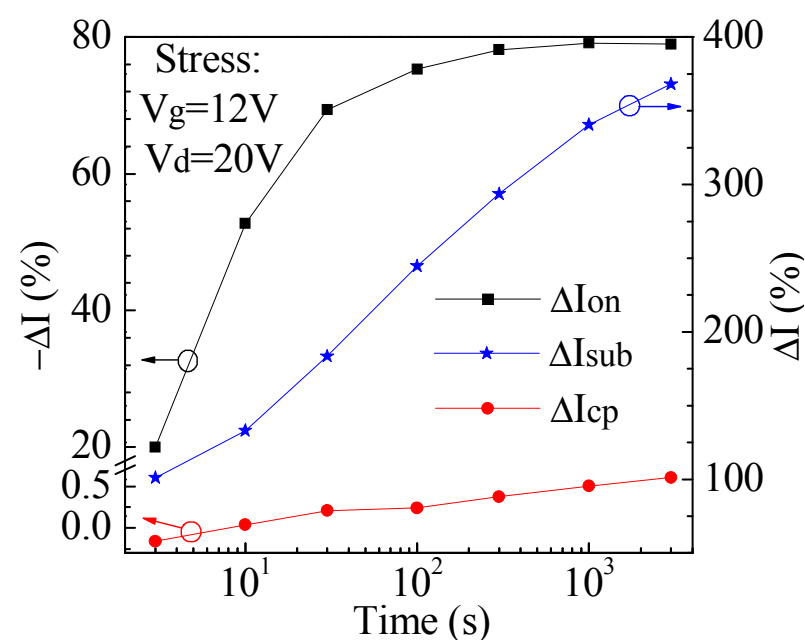

Fig.10. The time dependence of the $\Delta I_{c p}$ obtained from the CP curves of Fig.9 with $V_{g b}=-12 \mathrm{~V}$, the $\Delta I_{s u b}$ extracted from the $I_{s u b, d^{-}} V_{g}$ curves of Fig. 6 with $V_{g}=-8 \mathrm{~V}$ and $\Delta I_{o n}$ determined from transfer curves in Fig.5 with $V_{g}=15 \mathrm{~V}$.

degradation [14], because the $\mathrm{CP}$ is only sensitive to the $D_{t}$ of the whole channel [12] while the HC-induced $D_{t}$ increase occurs locally in the D junction.

On the contrary, such local $D_{t}$ increase can be estimated from the time evolution of $I_{s u b, d}$ with $V_{s u b}=0.07 \mathrm{~V}$ in Fig.8. As mentioned above, in high $-V_{g}$ range (e.g., $\left.-8 \mathrm{~V}\right), I_{\text {sub,d }}$ derives from the recombination process in the $\mathrm{D}$ junction and therefore is in accordance with the $D_{t}$ in the $\mathrm{HC}$ damage region near the drain terminal, according to Eq.2. As shown Fig.10, the obvious increment of $I_{s u b, d}$ with $V_{s u b}=0.07 \mathrm{~V}$ and $V_{g}=-8 \mathrm{~V}$ reflects a notably $D_{t}$ increase in the $\mathrm{HC}$ damage region, which will form a high barrier near the drain and therefore dramatically reduce $I_{o n}$ [11]. This is well consistent with the dramatic $I_{o n}$ degradation show in Fig. 10.

\section{SUMMARY}

The mechanism of $I_{s u b}$ in poly-Si TFTs is first clarified. $I_{s u b}$ is found to be a diode of a gated PIN Structure and $I_{s u b}$ at low $V_{s u b}$ is driven by the recombination process in the channel region. Besides, under the control of $V_{g}$, the recombination area locates at the whole channel or the local area near drain, source or substrate terminal. Based on this insight, the $I_{\text {sub }}-V_{g}$ curve together with its two component, i.e., the $I_{s u b, d^{-}} V_{g}$ and $I_{s u b, s^{-}} V_{g}$ curves is applied to investigate the $\mathrm{HC}$ degradation of poly-Si TFTs. Not only is the HC damage region accurately located, but also the HC-induced $D_{t}$ increase near the drain is sensitively detected, which easily overcomes the deficiency of the $\mathrm{CP}$ on the $\mathrm{HC}$ investigation.

\section{ACKNOWLEDGMENT}

This work was supported by the Natural Science Foundation of Jiangsu Province of China (BK2009112) and the National Natural Science Foundation of China (60406001).

\section{REFERENCES}

[1] Y. Nakajima, Y. Kida, M. Murase, Y. Toyoshima, and Y. Maki, "Latest developments for 'system-on-glass' displays using low-temperature poly-Si TFTs,” J. Soc. Inf. Disp., vol. 12, no. 4, pp. 361-365, 2004.

[2] Hu C., Tam S.C., Hsu F.C., Ko P.K., Chan T.Y. and Terrill K.W., "Hot-electron-induced MOSFET degradation-Model, monitor, and improvement," IEEE Trans. on Electron Devices, vol. 32, pp. 375-85, 1985.

[3] N.A. Hastas, Archontas N., Dimitriadis C. A., Kamarinos G., Nikolaidis T., Georgoulas N. and Thanailakis A, "Substrate current and degradation of n-channel polycrystalline silicon thin-film transistors," Microelectronics Reliability, vol. 41, pp. 341-8, 2005.

[4] M. A. Kroon, Van Swaaij and R. A. C. M. M., "Spatial effects on ideality factor of amorphous silicon pin diodes," Journal of Applied Physics, vol. 90, pp. 994-1000, 2001.

[5] Mark Stewart and Miltiadis K. Hatalis, "High performance gated lateral polysilicon PIN diodes," Solid-State Electron., vol. 44, pp. 1613-9, 2000.

[6] Camara N., Bano E. and Zekentes K., "Current transport mechanisms in 4H-SiC pin diodes," 2003 International Semiconductor Conference. CAS 2003 Proceedings (IEEE Cat. No. 03TH8676), vol. 2, pp. 249-52, 2003.

[7] Ruzin A. And Marunko S., "Current mechanisms in silicon PIN structures processed with various technologies," Nuclear Instruments \& Methods in Physics Research, Section A (Accelerators, Spectrometers, Detectors and Associated Equipment), vol. 492, no. 3, pp. 411-22, 2002.

[8] Badila M., Tudor B., Brezeanu G., Locatelli M.L., Chante J.P., Millan J., Godignon P., Lebedev A. and Banu, V., "Current-voltage characteristics of large area $6 \mathrm{H}-\mathrm{SiC}$ pin diodes," Materials Science \& Engineering B (Solid-State Materials for Advanced Technology), vol. B61-B62, pp. 433-6, 1999.

[9] Sooraj V. Karnik and Miltiadis K. Hatalis, "Lateral polysilicon $\mathrm{p}^{+}-\mathrm{p}-\mathrm{n}^{+}$and $\mathrm{p}^{+}-\mathrm{n}-\mathrm{n}^{+}$diodes," Solid-State Electron., vol. 47, pp. 653-9, 2003.

[10] C. Van Berkel, Powell M.J., Franklin A.R. and French I.D., "Quality factor in a-SiH nip and pin diodes," Journal of Applied Physics, vol. 73, pp. 5264-8, 1993.

[11] Min Xue, Mingxiang Wang, Zhen Zhu, Dongli Zhang and Man Wong, "Degradation behaviors of metal-induced laterally crystallized n-type polycrystalline silicon thin-film transistors under DC bias stresses," IEEE Trans. on Electron Devices, vol. 54, pp. 225-32, 2007.

[12] G. Groeseneken, H.E. Maes, N. Beltran, R. F. De Keersmaecker, "A Reliable Approach to Charge-Pumping Measurements in MOS Transistors," IEEE Transactions on Electron Devices, vol. ED-31, pp. 42-53, 1984.

[13] Lei LU, Mingxiang Wang and Man Wong, "Geometric effect elimination and reliable trap state density extraction in charge pumping of polysilicon thin-film transistors," IEEE Electron Device Letters, vol. 30, pp. 517-9, 2009.

[14] Xiaowei Lu, Mingxiang Wang, Kai SUN, and Lei LU, "Evaluation of Self-Heating and Hot Carrier Degradation of Poly-Si Thin-Film Transistors using Charge Pumping technique," 2010 IEEE International Reliability Physics Symposium, pp. 1040-1043, 2010. 